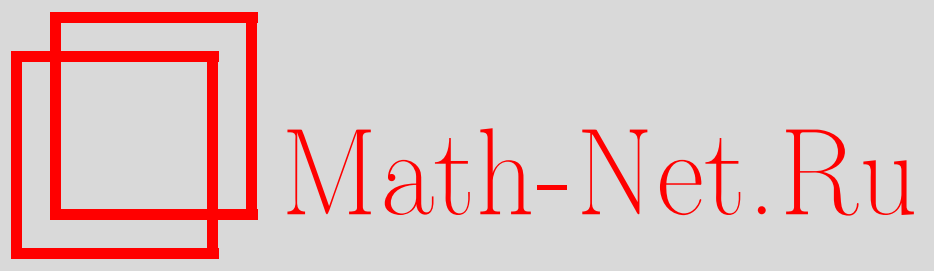

С. В. Козырев, Многомерная кластеризация и гиперграфы, $T M \Phi, 2010$, том 164, номер 3, 394-400

DOI: https://doi.org/10.4213/tmf6549

Использование Общероссийского математического портала Math-Net.Ru подразумевает, что вы прочитали и согласны с пользовательским соглашением http: //www.mathnet.ru/rus/agreement

Параметры загрузки:

IP: 54.224 .187 .69

26 апреля 2023 г., 13:55:52

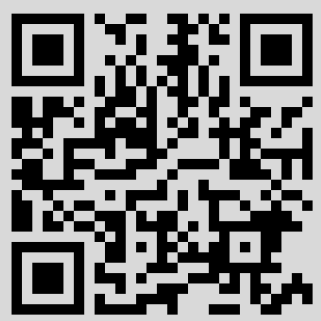




\title{
МНОГОМЕРНАЯ КЛАСТЕРИЗАЦИЯ И ГИПЕРГРАФЫ
}

\begin{abstract}
Обсуждается многомерное обобщение метода кластеризации. В рассматриваемом подходе кластеризация осуществляется частично упорядоченными гиперграфами из некоторого семейства. Процедура применима к случаю, когда исходная метрика зависит от набора параметров. Изучаемый здесь гиперграф кластеризации можно рассматривать как объект, описывающий всевозможные деревья кластеризации, отвечающие различным значениям исходной метрики.
\end{abstract}

Ключевые слова: кластеризация, гиперграф, анализ данных.

\section{1. ОБСУЖДЕНИЕ И ПОСТАНОВКА ЗАДАЧИ}

В настоящей работе мы обсудим кластеризацию (см. монографию [1]) и иерархические структуры, применяемые в анализе данных.

Кластеризация есть метод приближения метрики ультраметрикой: если на множестве задана некоторая метрика, то мы можем построить приближающую ее ультраметрику как цепное расстояние (см. определения 2, 3). Такое расстояние удовлетворяет всем аксиомам ультраметрики, кроме невырожденности; для некоторых множеств (в частности, для дискретных) оно удовлетворяет также аксиоме невырожденности. В разделе 4 мы обсудим процедуру кластеризации, а в разделе 3 связи деревьев и ультраметрических пространств.

Шары относительно построенной таким образом ультраметрики называются кластерами, процедура построения ультраметрики - процедурой кластеризации (мы привели простейший пример такой процедуры, возможны некоторые модификации). Если построенная ультраметрика не является невырожденной (т. е. расстояние между некоторыми нетождественными точками равно нулю), то точки с нулевым расстоянием между ними отождествляются.

С такой процедурой связана известная проблема. А именно исходная метрика, к которой применяется кластеризация, может быть определена неоднозначным образом - метрика может зависеть от набора параметров (весов модели). В зависимости от значений этих параметров у нас будут возникать при кластеризации различные ультраметрические пространства и различные отвечающие им частично упорядоченные деревья кластеров.

* Математический институт им. В. А. Стеклова РАН, Москва, Россия. E-mail: kozyrev@mi.ras.ru 
Обычный способ решения этой проблемы следующий. Считается, что можно зафиксировать некоторым образом значения параметров, от которых зависит метрика, - выбрать правильные параметры, которые дадут правильное дерево кластеров.

Нам представляется, что это не единственно возможная и даже не наиболее естественная точка зрения. В настоящей работе мы предлагаем рассматривать вместо одного дерева кластеризации, отвечающего выбранному значению параметров метрики, объединение деревьев, отвечающих разным значениям параметров. Такое объединение уже не будет деревом и будет содержать циклы. Мы покажем, что такие циклы естественно интерпретировать как ребра более высокого порядка, и граф кластеризации считать гиперграфом. В частности, рассмотрим случай, когда изучаемая система является прямым произведением подсистем, каждая из которых описывается некоторой метрикой; соответственно вся система описывается прямым произведением метрик. Процедура кластеризации на каждой из подсистем даст свое дерево кластеров. Естественно ожидать (если мы хотим учесть независимость подсистем), что вся система должна описываться произведением деревьев кластеров подсистем. Но произведение деревьев не будет деревом и даже графом - произведение деревьев является гиперграфом. В свою очередь кластеризация всей системы по метрике прямого произведения даст некоторое дерево, в котором не будет заметна в явном виде структура прямого произведения деревьев подсистем.

Напомним, что гиперграфом называется некоторое множество $\Gamma$, на котором выделена система конечных подмножеств $E$, состоящая из подмножеств, содержащих два или более элементов Г. Элементы множества Г называются вершинами гиперграфа, конечные подмножества (набора вершин гиперграфа) из $E$ - ребрами гиперграфа. В том случае, когда все ребра из $E$ имеют мощность два, гиперграф называется графом.

Прямое произведение двух графов $\left(\Gamma_{1}, E_{1}\right)$ и $\left(\Gamma_{2}, E_{2}\right)$ будет гиперграфом с множеством вершин $\Gamma_{1} \times \Gamma_{2}$ и ребрами порядка 2 и 4 следующего вида. Пусть в первом и втором графах соответственно есть ребра $\left(A_{1}, B_{1}\right)$ и $\left(A_{2}, B_{2}\right)$. Этой паре ребер мы сопоставим четыре 2-ребра гиперграфа-произведения, являющиеся строками и столбцами $(2 \times 2)$-матрицы

$$
\left(\begin{array}{cc}
A_{1} \times A_{2} & A_{1} \times B_{2} \\
B_{1} \times A_{2} & B_{1} \times B_{2}
\end{array}\right) .
$$

Набор всех элементов этой матрицы будет 4-ребром. Мы определяем набор ребер гиперграфа-произведения при помощи описанной процедуры; 2-ребра - произведения вершин одного графа на ребра другого, 4-ребра - произведения ребер графов-сомножителей.

Целью настоящей работы является обсуждение процедуры кластеризации, которая воспроизводила бы описанный выше гиперграф-произведение, а также обобщения такого гиперграфа на случай, когда исходная метрика не является прямым произведением.

Таким образом, стартовой точкой для нас являются следующие два наблюдения.

1. Тензорная структура (структура произведения, структура независимости) на исходных данных не воспроизводится методами кластеризации. Хотелось бы иметь модификацию методов кластеризации, которая в некоторой степени воспроизводила 
бы такую структуру. Естественным объектом для этого является кластеризация в гиперграфах.

2. Дерево кластеров в случае, когда метрика зависит от набора параметров, строится не единственным образом, т. е. фактически мы имеем семейство деревьев кластеров. Хотелось бы сопоставить это семейство деревьев упомянутой выше процедуре кластеризации гиперграфами - независимые параметры в исходной метрике, вызывающие перестройки дерева кластеров, сопоставить структуре произведения на гиперграфах.

В следующем разделе мы обсудим два примера многомерной кластеризации гиперграфами: для случая, когда гиперграф не имеет структуры произведения деревьев, и для случая, когда такая структура имеет место.

\section{2. ПРОСТЕЙШИЕ ПРИМЕРЫ}

Пример 1. Случай множества из трех точек. Такие точки можно рассматривать, например, как точки двумерной вещественной плоскости $\mathbb{R}^{2}$ со стандартной метрикой. Параметры, от которых зависит метрика, - координаты точек плоскости. Для точек $A, B, C$ имеем набор кластеров, например $A, B, C, A B, A B C$. Эти кластеры суть вершины дерева кластеров, ребра соединяют вершины в соответствии с ростом кластеров, т. е. данное дерево кластеров содержит ребра $(A, A B),(B, A B)$, $(A B, A B C),(C, A B C)$.

Перестройка кластеров при изменении метрики (движении точек на плоскости $\mathbb{R}^{2}$ ) может выглядеть как замена описанного выше набора кластеров на набор $A, B, C$, $B C, A B C$ с соответствующими ребрами $(B, B C),(C, B C),(B C, A B C),(A, A B C)$.

Каждому из таких наборов кластеров отвечает свое дерево кластеров. Мы рассматриваем гиперграф с множеством вершин, являющимся объединением множеств вершин деревьев (т. е. содержащим $A, B, C, A B, B C, A B C$ ), набором 2-ребер (ребер из двух точек), являющимся объединением наборов 2-ребер из двух описанных деревьев, и следующими ребрами более высокого порядка: 3 -ребрами $(A, A B, A B C)$, $(C, B C, A B C)$ и 4-ребром $(B, A B, B C, A B C)$.

Таким образом, ребра более высокого порядка описывают рост кластера (в обоих деревьях кластеров, которые мы объединяем в одном гиперграфе), начиная с некоторой вершины. Ребра высокого порядка отвечают циклам в графе - объединении деревьев, отвечающих кластеризациям при разных значениях параметров.

Пример 2. Случай множества из четырех точек $A, B, C, D$, которые мы считаем расположенными на плоскости $\mathbb{R}^{2}$ в вершинах некоторого четырехугольника. В этом четырехугольнике, используя кластеризацию по метрике плоскости, мы выделяем следующие кластеры: $A, B, C, D, A B, C D, A B C D$, т. е. мы рассматриваем не все возможные для случая общего положения кластеры, а только часть, как получается, например, в процедуре кластеризации по шкале расстояний (см. раздел 4). Множество 2-ребер будет содержать ребра $(A, A B),(B, A B),(C, C D),(D, C D)$, $(A B, A B C D),(C D, A B C D)$.

Рассмотрим теперь деформацию четырехугольника (например, растяжение вдоль некоторого направления на плоскости $\mathbb{R}^{2}$ ), при котором дерево кластеров принимает следующий вид: дерево будет содержать вершины $A, B, C, D, A C, B D, A B C D$ и 2-ребра $(A, A C),(C, A C),(B, B D),(D, B D),(A C, A B C D),(B D, A B C D)$. 
Из этих двух деревьев мы строим гиперграф, содержащий объединение множеств вершин и 2-ребер из описанных деревьев, а также 4-ребра $(A, A B, A C, A B C D)$, $(B, A B, B D, A B C D),(C, A C, C D, A B C D),(D, B D, C D, A B C D)$.

Схематично такой гиперграф можно изобразить следующей матрицей:

$$
\left(\begin{array}{ccc}
A & A C & C \\
A B & A B C D & C D \\
B & B D & D
\end{array}\right)
$$

Здесь матричные элементы суть вершины гиперграфа, 2-ребра соединяют соседние (по горизонтали и вертикали) вершины, 4-ребра отвечают $(2 \times 2)$-квадратикам, содержащим углы матрицы.

Аналогичная матрица для примера 1 будет иметь вид половины приведенной выше матрицы:

$$
\left(\begin{array}{ccc}
A & A C & C \\
A B & A B C & \\
B & &
\end{array}\right)
$$

ребра будут ограничениями соответствующих ребер.

Полученный в примере 2 гиперграф можно рассматривать как произведение двух деревьев, отвечающих независимым кластеризациям пар точек на двух вещественных прямых: дерева $\mathcal{T}_{1}$ с вершинами $x_{1}, y_{1}, x_{1} y_{1}$ и ребрами $\left(x_{1}, x_{1} y_{1}\right),\left(y_{1}, x_{1} y_{1}\right)$ и дерева $\mathcal{T}_{2}$ с вершинами $x_{2}, y_{2}, x_{2} y_{2}$ и ребрами $\left(x_{2}, x_{2} y_{2}\right),\left(y_{2}, x_{2} y_{2}\right)$.

Тогда можно рассматривать вершины $A=x_{1} \times y_{1}, B=x_{2} \times y_{1}, C=x_{1} \times y_{2}$, $D=x_{2} \times y_{2}$ как произведения вершин из $\mathcal{T}_{1}, \mathcal{T}_{2}$. Остальные вершины гиперграфа будут (как кластеры) их объединениями, например $A B=\left\{x_{1} \times y_{1}, x_{2} \times y_{1}\right\}, A C=$ $\left\{x_{1} \times y_{1}, x_{1} \times y_{2}\right\}$.

2-Ребра гиперграфа будут отвечать ребрам одного из этих деревьев, умноженным на вершину другого дерева. Например, ребро $(A, A B)$ есть

$$
\left(x_{1} \times y_{1},\left\{x_{1} \times y_{1}, x_{2} \times y_{1}\right\}\right)=\left(x_{1}, x_{1} x_{2}\right) \times y_{1} \text {. }
$$

4-Ребра гиперграфа отвечают произведениям двух ребер деревьев-сомножителей. Например, 4-ребро $(A, A B, A C, A B C D)$ имеет вид

$$
\begin{aligned}
& \left(x_{1} \times y_{1},\left\{x_{1} \times y_{1}, x_{2} \times y_{1}\right\},\left\{x_{1} \times y_{1}, x_{1} \times y_{2}\right\},\left\{x_{1} \times y_{1}, x_{2} \times y_{1}, x_{1} \times y_{2}, x_{2} \times y_{2}\right\}\right)= \\
& \quad=\left(x_{1}, x_{1} x_{2}\right) \times\left(y_{1}, y_{1} y_{2}\right) .
\end{aligned}
$$

Изображение гиперграфа матрицей можно представить в виде матрицы произведений:

$$
\left(\begin{array}{ccc}
x_{1} \times y_{1} & x_{1} \times\left(y_{1}, y_{2}\right) & x_{1} \times y_{2} \\
\left(x_{1}, x_{2}\right) \times y_{1} & \left(x_{1}, x_{2}\right) \times\left(y_{1}, y_{2}\right) & \left(x_{1}, x_{2}\right) \times y_{2} \\
x_{2} \times y_{1} & x_{2} \times\left(y_{1}, y_{2}\right) & x_{2} \times y_{2}
\end{array}\right) .
$$

Таким образом, гиперграф, получаемый в данном частном случае перестройки деревьев, можно представить в виде произведения двух деревьев $\mathcal{T}_{1}, \mathcal{T}_{2}$, что отражает внутреннюю двумерную структуру данных. 
На всех построенных гиперграфах (точнее, на их множествах вершин) присутствует частичный порядок (отвечающий вложению меньших кластеров в бо́льшие). При этом все 2-ребра будут ориентированы, т. е. один конец ребра всегда будет сравним с другим.

В общем случае процедура многомерной кластеризации состоит в следующем.

1. Построение деревьев кластеров для различных значений параметров, от которых зависит метрика.

2. Взятие объединений таких деревьев, результатом чего будет частично упорядоченный граф, не являющийся в общем случае деревом, но не содержащий ориентированных (упорядоченных) циклов.

3. Анализ структуры такого графа, позволяющий представить его как гиперграф, т. е. построение ребер порядка выше 2. Как 2-ребра графа кластеризации описывают вложения кластера в более крупный кластер, так ребра более высокого порядка будут описывать историю таких вложений относительно различных значений параметров метрики. В рассмотренных примерах 3-ребра и 4-ребра отвечали циклам в графе-объединении, построенном на втором шаге процедуры. Такие 3и 4-ребра являются двумерными объектами, что видно из обсуждения примера 2. В случае объектов более высокой размерности будут появляться ребра более высокого порядка, отвечающие произведениям некоторого числа вершин и 2-ребер, а также их подмножествам.

4. Дальнейший анализ структуры гиперграфа должен выявить структуру произведений для некоторых наборов ребер различного порядка.

\section{3. УЛЬТРАМЕТРИЧЕСКИЕ ПРОСТРАНСТВА И ДЕРЕВЬЯ}

Ультраметрическое пространство есть метрическое пространство, где выполнено сильное неравенство треугольника.

ОПРеДЕЛЕНИЕ 1. Ультраметрическое пространство $X$ есть множество с ультраметрикой $d(x, y)$ (где $d(x, y)$ называется расстоянием между точками $x$ и у из $X)$, т. е. функцией двух переменных, удовлетворяющей свойствам положительности и невырожденности:

$$
d(x, y) \geqslant 0, \quad d(x, y)=0 \Leftrightarrow x=y \quad \forall x, y
$$

симметричности:

$$
d(x, y)=d(y, x) \quad \forall x, y,
$$

и сильному неравенству треугольника:

$$
d(x, y) \leqslant \max (d(x, z), d(y, z)) \quad \forall x, y, z .
$$

Шаром называется множество всех точек, удаленных от некоторой точки (центра шара) на расстояние не более заданного (радиус шара). Диаметром множества называется точная верхняя грань для расстояний между точками этого множества.

Ультраметрические пространства двойственны к некоторым частично упорядоченным деревьям. Опишем часть конструкции двойственности.

Для (полного локально-компактного) ультраметрического пространства $X$ рассмотрим множество $\mathcal{T}(X)$, содержащее все шары в $X$ ненулевого диаметра, а также 
шары нулевого диаметра, являющиеся максимальными подшарами в шарах нулевого диаметра. Такое множество $\mathcal{T}(X)$ имеет естественную структуру частично упорядоченного множества, задаваемую вложением шаров друг в друга. Множество $\mathcal{T}(X)$ мы будем считать деревом, вершинами которого являются описанные шары в $X$. Два шара $I$ и $J$ соединяются ребром, если один является максимальным подшаром другого (т. е. между этими двумя шарами нет промежуточных шаров).

На частично упорядоченном дереве $\mathcal{T}(X)$ рассмотрим возрастающую положительнозначную функцию, ставящую шару в соответствие его диаметр.

Обратно, пусть мы имеем частично упорядоченное дерево $\mathcal{T}$ с не более чем счетным числом вершин, удовлетворяющее следующим условиям:

1) граф $\mathcal{T}$ есть дерево, т. е. для любой пары вершин существует единственный конечный путь (без самопересечений), соединяющий эти вершины;

2) каждая вершина из $\mathcal{T}$ принадлежит конечному множеству ребер;

3) каждый конечный путь в $\mathcal{T}$ содержит единственную максимальную вершину.

Выберем произвольную положительную возрастающую (относительно частичного порядка) функцию $F$ на дереве $\mathcal{T}$. Тогда функция

$$
d(I, J)=F(\sup (I, J)), \quad I \neq J, \quad d(I, I)=0,
$$

где $\sup (I, J)$ есть точная верхняя грань пары вершин $I, J$ по отношению к частичному порядку, будет ультраметрикой на $\mathcal{T}$. Вершина $\sup (I, J)$ совпадает с единственной максимальной вершиной на пути $I J$.

Рассмотрим пополнение $\mathcal{T}$ относительно введенной ультраметрики и удалим из пополнения все внутренние точки дерева $\mathcal{T}$ (вершина является внутренней, если она не принадлежит границе дерева, т. е. принадлежит более чем одному ребру). Полученное пространство $X(\mathcal{T})$ будет ультраметрическим, полным и локально-компактным.

\section{4. КЛАСТЕРИЗАЦИЯ}

Обсудим процедуру кластеризации для метрических пространств. Обозначим через $(M, \rho)$ метрическое пространство $M$ с метрикой $\rho$.

ОПРЕДЕЛЕНИЕ 2. Последовательность точек $a=x_{0}, x_{1}, \ldots, x_{n-1}, x_{n}=b$ в метрическом пространстве $(M, \rho)$ называется $\varepsilon$-цепью, соединяющей точки $a$ и $b$, если $\rho\left(x_{k}, x_{k+1}\right) \leqslant \varepsilon$ для всех $0 \leqslant k<n$. Если существует $\varepsilon$-цепь, соединяющая $a$ и $b$, то говорят, что $a$ и $b$-связываемы.

В ультраметрическом пространстве любые две точки $a$ и $b$ не $\varepsilon$-связываемы для $\varepsilon<\rho(a, b)$.

ОПРЕДЕЛЕНИЕ 3 . Пусть $(M, \rho)$ есть произвольное метрическое пространство. Тогда цепное расстояние $d(a, b)=\inf (\varepsilon: a, b$-связываемы).

Цепное расстояние $d(a, b)$ между точками $a$ и $b$ обладает всеми свойствами ультраметрики, кроме невырожденности, т. е.

$$
\begin{aligned}
& d(a, b)=d(b, a) \quad \forall a, b, \\
& d(a, b) \leqslant \max (d(a, c), d(c, b)) \quad \forall a, b, c,
\end{aligned}
$$

но для некоторых $a \neq b$ может быть $d(a, b)=0$. 
В некоторых случаях (например, если пространство $(M, \rho)$ дискретно) цепное расстояние будет невырожденно и, следовательно, будет ультраметрикой. Если исходное пространство $(M, \rho)$ ультраметрично, то цепное расстояние совпадает с исходной ультраметрикой $d(a, b)=\rho(a, b)$.

ОПРЕДЕЛЕНИЕ 4. Назовем кластером $C(i, R)$ в метрическом пространстве $(M, \rho)$ шар относительно цепного расстояния с центром в $i$ радиуса $R$, т. е. множество $\{j \in$ $M: d(i, j) \leqslant R\}$. Кластеризацией пространства $M$ назовем набор кластеров в $M$ такой, что: каждый элемент из $M$ лежит в некотором кластере; для каждой пары $a, b$ элементов из $M$ существует минимальный содержащий их кластер $\sup (a, b)$; для любых вложенных кластеров $A \subset B$ любая возрастающая последовательность вложенных кластеров $\left\{A_{i}\right\}, A \subset \cdots \subset A_{i} \subset A_{i+1} \subset \cdots \subset B$ конечна.

Различные кластеризации составляют частично упорядоченное множество: если мы имеем две кластеризации $\mathcal{A}$ и $\mathcal{B}$, то $\mathcal{A}>\mathcal{B}$ в случае, когда все кластеры из $\mathcal{B}$ содержатся в $\mathcal{A}$.

Поскольку цепное расстояние удовлетворяет сильному неравенству треугольника, кластеризация $\mathcal{C}$ порождает частично упорядоченное дерево кластеров $\mathcal{T}=\mathcal{T}(M)$ и ультраметрику на этом дереве (цепное расстояние между кластерами). Далее, по стандартной процедуре мы строим ультраметрическое пространство $X=X(\mathcal{T})$ (цепное пространство кластеризации $\mathcal{C}$ ), отождествляемое с границей дерева $\mathcal{T}$.

Кластеры в пространстве $M$ соответствуют шарам в пространстве $X$. Кластеризация позволяет грубо описать метрическое пространство $(M, \rho)$ ультраметрическим пространством $(X, d)$.

Пример 3. Пусть $D=\left\{d_{i}\right\}$ есть счетный набор положительных чисел, не имеющий точек сгущения, кроме, может быть, нуля, и не ограниченный сверху. Такой набор будем называть шкалой расстояний. Тогда кластеризация $\mathcal{C}_{D}$ метрического пространства $(M, \rho)$ содержит всевозможные кластеры цепных радиусов $d_{i} \in D$.

Такую кластеризацию мы будем называть кластеризацией по шкале расстояний $D$.

Благодарности. Автор благодарен И. В. Воловичу за плодотворные обсуждения. Работа была частично поддержана грантами DFG Project 436 RUS 113/809/0-1 и DFG Project 436 RUS 113/951, грантами РФФИ № 08-01-00727-а и 09-01-12161офи_м, грантом президента РФ поддержки научных школ НШ-7675.2010.1 и программой Отделения математики РАН "Современные проблемы теоретической математики", а также аналитической ведомственной целевой программой МОН "Развитие научного потенциала высшей школы" на 2009-2010 гг. (проект № 3341).

\section{Список литературы}

[1] F. Murtagh, Multidimensional Clustering Algorithms, Compstat Lectures, 4, Physica, Heidelberg, 1985. 